\title{
Optical switching in midinfrared light-emitting diodes
}

 \\ Physics Department, Lancaster University, Lancaster LA1 4YB, United Kingdom
}

A. M. Monahov

Ioffe Physico-Technical Institute, St Petersburg, 195279, Russia

G. Hill

III-V Central Facility, Electronic \& Electrical Engineering Department, University of Sheffield, Mappin St. Sheffield, S1 3JD, United Kingdom

(Received 19 November 2001; accepted for publication 28 February 2002)

We report on the optical quenching of electroluminescence in midinfrared light-emitting diodes operating at $3.0 \mu \mathrm{m}$. The source is based on a symmetrical double heterostructure with large band offsets and is effectively switched off using coherent visible light. (C) 2002 American Institute of Physics. [DOI: 10.1063/1.1470690]

Many types of optical switching devices ${ }^{1-4}$ have been studied and demonstrated using nonlinear effects in a variety of semiconductor materials and more recently in optical fibers. Light-controlled switches have a wide variety of potential applications in optical communications and high-speed optoelectronic systems. Optically controlled, fiber-based switches such as loop mirrors or soliton gates can be very fast with good contrast ratios, low-power, and cascadable Boolean logic, but they are difficult to arrange in arrays and integrate with electronics. ${ }^{5-8}$ Although such components are commonplace in the visible and near infrared $(<1.55 \mu \mathrm{m})$, there have to date been inadequate reports of optical switching in the midinfrared spectral region $(2-5 \mu \mathrm{m})$. In this letter, we report on the optical quenching of midinfrared electroluminescence at $3.0 \mu \mathrm{m}$, which could form the basis of a midinfrared optical switch

The light-emitting diode (LEDs) were fabricated from III-V double heterostructures (DHs) grown by liquid phase epitaxy (LPE). A conventional horizontal, graphite sliding boat was used for the LPE growth of the LED structures onto $p$-type InAs (100) substrates. These were $14 \mathrm{~mm} \times 14 \mathrm{~mm}$ square with a carrier concentration of $\sim 1 \times 10^{18} \mathrm{~cm}^{-3}$ and were obtained from Wafer Technology Ltd. Epitaxy was carried out with the graphite boat inside a high purity quartz reactor tube under flowing ultrapure hydrogen gas provided from a Pd-diffusion unit. The apparatus was fully automated and controlled with LABVIEW operating software from a personal computer. The resulting epitaxial structure, which has been described in detail previously elsewhere, ${ }^{9}$ was a $\mathrm{DH}$ in which the unintentionally doped $n$-InAs active layer is enclosed between $p$ - and $n$ - $\operatorname{InAs}_{0.42} \mathrm{Sb}_{0.18} \mathrm{P}_{0.40}$ confinement layers. The $\mathrm{P}$ content in the confinement layers was $0.40\left(E_{g}\right.$ $=640 \mathrm{meV}$ and $T=4 \mathrm{~K}$ ) to provide large interface band offsets $\Delta E_{c}=151 \mathrm{meV}$ and $\Delta E_{v}=21 \mathrm{meV}$ for good carrier confinement. The InAs active region $\left(E_{g}=415 \mathrm{meV}\right.$ and $T$ $=4 \mathrm{~K})$ was $0.7 \mu \mathrm{m}$ thick, and the $\operatorname{InAs}_{0.42} \mathrm{Sb}_{0.18} \mathrm{P}_{0.40}$ layers were isoperiodic with InAs, each being $3.0 \mu \mathrm{m}$ in thickness. $\mathrm{By}$ using $\mathrm{Yb}$ rare-earth gettering, the residual carrier concen-

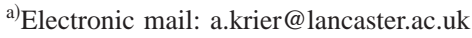

tration in the active layer was reduced to $<5 \times 10^{15} \mathrm{~cm}^{-3}$. The cladding layers were intentionally doped with $\mathrm{Sn}$ up to a concentration of $5 \times 10^{18} \mathrm{~cm}^{-3}$ and with $\mathrm{Zn}$ up to 1 $\times 10^{18} \mathrm{~cm}^{-3}$ for $n$ - and $p$-sides, respectively.

LEDs were fabricated from the epitaxial wafers using standard procedures by employing conventional photolithography and reactive ion etching with $\mathrm{CH}_{4}: \mathrm{H}_{2}$ to produce mesas $420 \mu \mathrm{m}$ in diameter with an emitting area of 1.39 $\times 10^{-3} \mathrm{~cm}^{2}$. After etching, the mesa was passivated with $\mathrm{Si}_{3} \mathrm{~N}_{4}$. Ohmic contacts were formed by thermal evaporation of Au:Zn and Au:Te alloys at $180^{\circ} \mathrm{C}$ on the $p$ - and $n$ sides of the structures, respectively. A $300 \mu \mathrm{m}$ diameter ohmic ring contact pad (30 $\mu \mathrm{m}$ width) was defined on the $n$ In $\mathrm{As}_{0.42} \mathrm{Sb}_{0.18} \mathrm{P}_{0.40}$ while the corresponding back contact was deposited over the entire rear surface of the chip. The LED chips were then mounted $n$ side up onto TO- 49 headers for testing using In solder. The resulting devices were operated with a drive current of $300 \mathrm{~mA}$ using $50 \mu$ s pulses at a frequency of $10 \mathrm{kHz}$, (duty cycle $50 \%$ ). The $4 \mathrm{~K}$ electroluminescence emission spectra from the edge of the LEDs were measured and compared for different levels of external illumination. A schematic diagram of the resulting light emitting diodes is shown in Fig. 1.

To study the effect of optical radiation on the electroluminescence, the sample was illuminated with coherent radia-

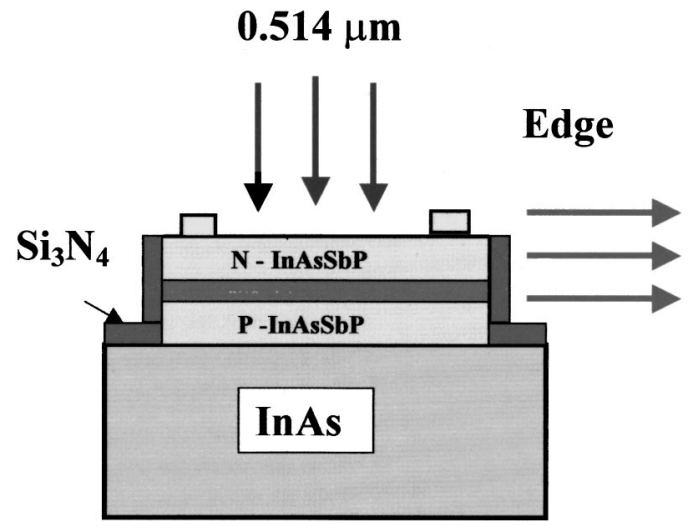

FIG. 1. (a) Schematic diagram of the DH LED showing the arrangement used in the experiment. 


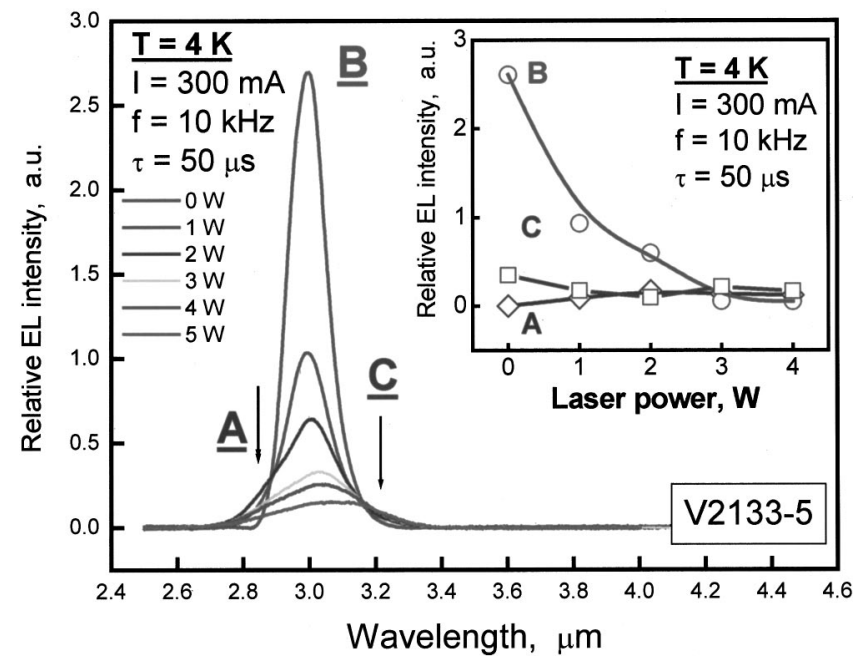

FIG. 2. $4 \mathrm{~K}$ electroluminescence emission spectra obtained from the edge of the LED measured with increasing optical illumination using a drive current of $300 \mathrm{~mA}$ at a frequency of $10 \mathrm{kHz}$ with $50 \mu$ s pulses. The inset shows the intensity dependence on power for each of the emission bands.

tion from a continuous wave argon ion laser (514 $\mathrm{nm}$ line) which was incident on the top of the LED mesa, but the focused spot size $(1 \mathrm{~mm})$ was larger than the mesa $(400 \mu \mathrm{m}$ diameter). The resulting emission was measured from the edge of the sample. The laser output power was varied from 0 to $5 \mathrm{~W}$, which represents a maximum optical excitation intensity at the sample of $\sim 80 \mathrm{~W} \mathrm{~cm}^{-2}$ inside the cryostat. The resulting electro- or photoluminescence (PL) was collected using $\mathrm{CaF}_{2}$ lenses and focused into a Bentham M300 monochromator. A cooled (77 K) InSb photodiode and Stanford Research (SR850) digital lock-in amplifier were used to detect the radiation. A computer was used to control the monochromator and record the output signal using LABVIEW operating software.

The results of the spectral measurements are shown in Figs. 2 and 3. It was found that the electroluminescence intensity decreased exponentially with increasing external illumination intensity. It was also noticed that the spectrum changed shape and became broader as the external illumination intensity increased. Careful examination of the spectrum obtained allows one to establish that without the external illumination the electroluminescence spectrum consists of two emission bands [B and $\mathrm{C}$ in Figs. 2 and 3(a)]. It is reasonable to attribute these bands to the emission originating from recombination between localized electrons and holes in quasi-quantum wells situated on opposite sides of the two heterointerfaces in the active region as shown in Fig. 4. The more intense band corresponds to transition B because the $p-n$ junction is situated right at this boundary. Deconvolution and analysis of the optical spectrum under external illumination using Gaussian curve fitting shows that three emission bands are now present in the electroluminescence as shown in Fig. 3(b). A new emission band (A) appears in the spectrum (see Figs. 2 and 3) under external illumination. We note that in a PL experiment on the LED structure a single broad band which does not change shape with small forward or reverse applied bias $(<0.1 \mathrm{~V})$ is observed. This broad band can be readily deconvoluted into the same bands A, B, and C observed in Figs. 2 and 3. We rule out heating
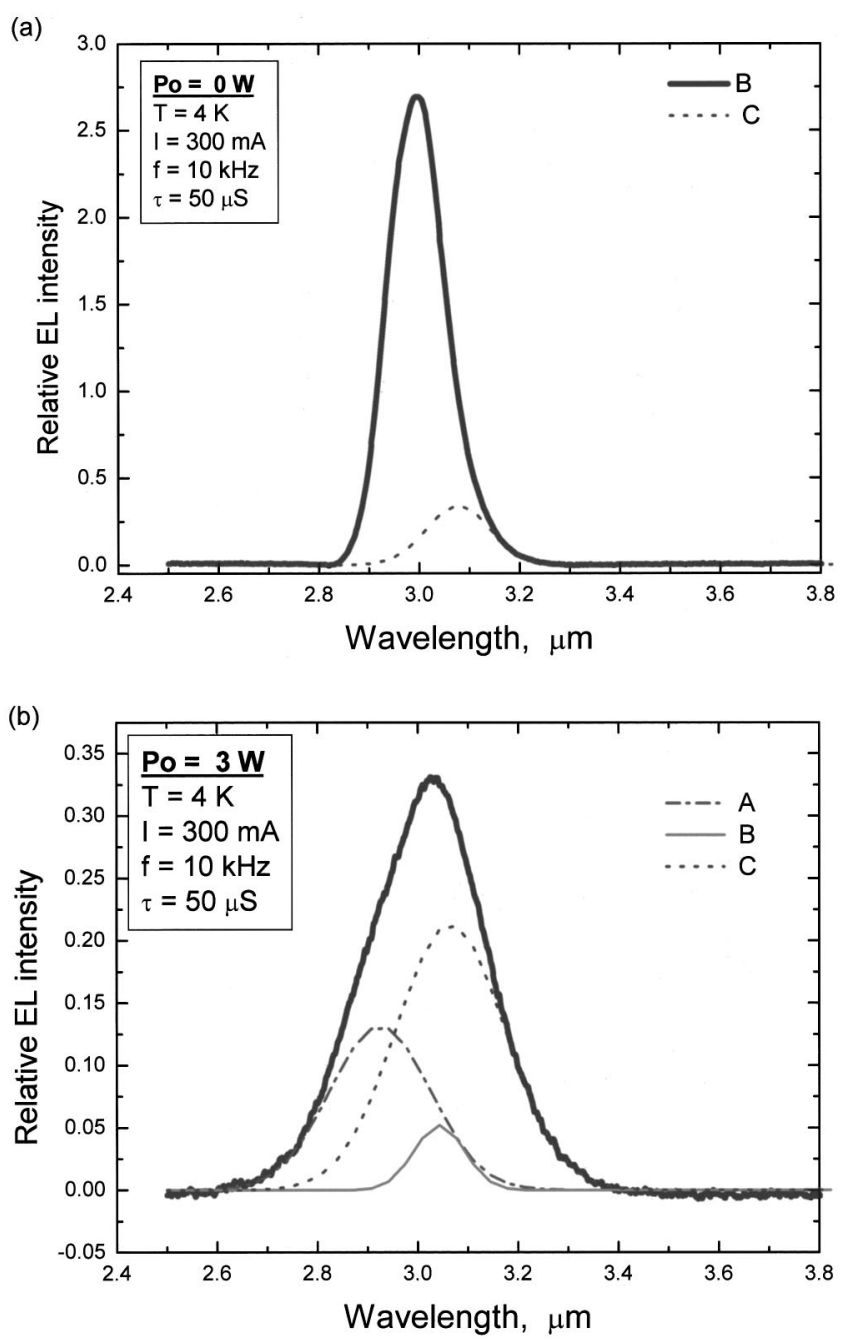

FIG. 3. Deconvolution of the $4 \mathrm{~K}$ electroluminescence spectra measured using the same drive conditions as in Fig. 2. (a) with zero external illumination, and (b) with an excitation of $3 \mathrm{~W}$.

from the laser as this should manifest itself as a significant redshift in peak wavelength corresponding to a band gap reduction and in our experiment, the principal peak remained at approximately constant wavelength. Furthermore, the magnitude of the quenching effect $(\times 25)$ which we observed is much too large to be of thermal origin.

The relative intensity of emission band $\mathrm{B}$ [Fig. 3(b)]



FIG. 4. Schematic energy band diagram of the DH LED structure under the influence of strong external illumination, showing the induced band bending and the associated radiative recombination. 


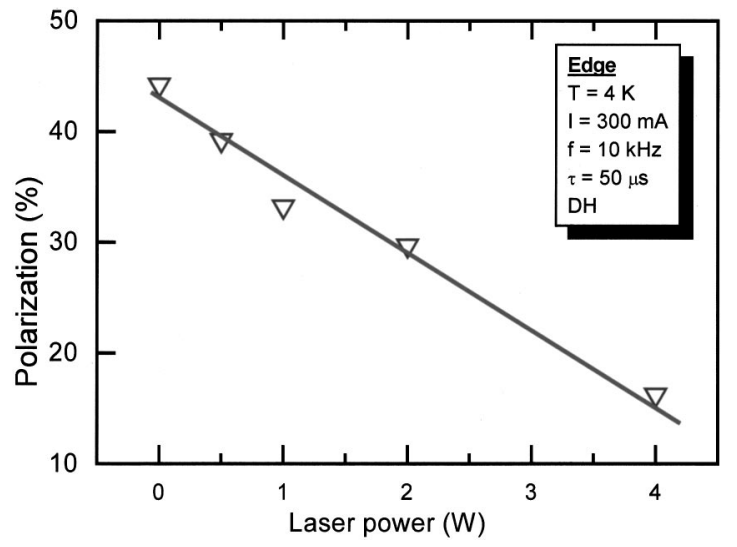

FIG. 5. Dependence of electroluminescence polarization on external illumination intensity measured from the edge of the LED at $4 \mathrm{~K}$.

becomes less than that of band $\mathrm{C}$ as the external illumination intensity is increased. In our opinion, the appearance of the band $\mathrm{A}$ is associated with the additional electron-hole pair production in the $n \operatorname{InAs}_{0.42} \mathrm{Sb}_{0.18} \mathrm{P}_{0.40}$ as a result of the intense external excitation. On application of the external illumination, electron-hole pairs are generated in the $n$ InAsSbP near the negative contact. The excess holes are driven toward the negative contact by the electric field and the electrons try and drift into the $p$ region. The high electron-hole density near the interface results in band bending which "traps" electrons in the optically induced potential well and so accounts for the appearance of the high energy emission band A. The quenching of band $\mathrm{B}$ relative to band $\mathrm{C}$ at the $n-\mathrm{N}$ type II heterojunction we attribute to the increased height of the potential barrier which restricts electron supply into the active region. With increasing illumination intensity, the bands B and C shifted towards the red region (from 2.989 to $3.045 \mu \mathrm{m}$ and from $3.070 \mu \mathrm{m}$ to $3.090 \mu \mathrm{m}$ for $\mathrm{B}$ and $\mathrm{C}$, respectively), whereas the band $\mathrm{A}$ shifted to the red (from 2.857 to $2.925 \mu \mathrm{m}$ ) and then back to the blue (2.925 to 2.918 $\mu \mathrm{m})$. Our findings are in accordance with the luminescence results from other materials including $\mathrm{Si}^{10,11} \mathrm{Ge},{ }^{12}$ and GaAs, ${ }^{13}$ where a similar phenomenon has been observed at the surface.

It should be mentioned that in this LED without the external illumination, optical amplification takes place due to the whispering gallery modes produced in the active region as we described in detail previously. ${ }^{14}$ Restricting the supply of electrons for recombination at B effectively spoils the gain of the whispering mode and so the intensity decreases rapidly. As shown in Fig. 5, we also measured the dependence of the degree of polarization of the total electroluminescence emission $p_{\|}-p_{\perp} / p_{\|}+p_{\perp}$ on the illumination intensity, and found that it falls linearly from $45 \%$ to $15 \%$. This is consistent with a strong reduction in the band $\mathrm{B}$ which is inherently polarized since it is a transition between quantum states on opposite sides of the heterointerface, whereas A is unpolar- ized. Alternatively, since the light emitted from the edge originates from a whispering mode which is itself polarized, the incident external illumination could produce a local reduction in refractive index in the InAs which reduces the effectiveness of the ring waveguide and results in depolarization of the light emitted from the edge of the LED.

The edge electroluminescence emission spectrum of a whispering gallery midinfrared LED has been investigated with and without external illumination using visible coherent light $(514 \mathrm{~nm})$. Without external illumination the spectra consists of two emission bands, which are due to type-II recombination at both heteroboundaries of the active region. Under strong external illumination, we observed depolarization and quenching of the LED emission and the appearance of a weak optically induced-interface emission band. The possibility of such optically induced band bending leading to new emission bands in type-II heterojunctions has been predicted, ${ }^{15}$ but until now has not been observed. The strong quenching which we observed was sufficient to effectively switch off the electroluminescence from the LED. Although demonstrated at low temperature and using high optical excitation, this result opens the opportunity for creation of midinfrared optical switches, modulators, and other lightcontrolled midinfrared devices.

The authors wish to thank EPSRC for the award of a research grant to support this work. One of the authors (A. M.) would like to thank the Ministry of Science of the Russian Federation for partial support of this work through the program "Optics and Laser Physics."

${ }^{1}$ Y. Nishikawa, A. Tackeuchi, S. Nakamura, S. Muto, and N. Yokoyama, Appl. Phys. Lett. 66, 839 (1995).

${ }^{2}$ R. Takahashi, Y. Kawamura, and H. Iwamura, Appl. Phys. Lett. 68, 153 (1996).

${ }^{3}$ T. Akiyama, M. Tsuchiya, and T. Kamiya, Appl. Phys. Lett. 72, 1545 (1998).

${ }^{4}$ R. Takahashi, H. Itoh, and H. Iwamura, Appl. Phys. Lett. 77, 2958 (2000).

${ }^{5}$ M. B. Yairi, C. W. Coldren, D. A. B. Miller, and J. S. Harris, Appl. Phys. Lett. 75, 597 (1999)

${ }^{6}$ M. N. Islam, C. E. Soccolich, and J. P. Gordon, Opt. Quantum Electron. 24, S1215 (1992).

${ }^{7}$ R. J. Manning, A. D. Ellis, A. J. Poustie, and K. J. Blow, J. Opt. Soc. Am. B 14, 3204 (1997).

${ }^{8}$ K. H. Ahn, X. D. Cao, Y. Liang, B. C. Barnett, S. Chaikamnerd, and M. N. Islam, J. Opt. Soc. Am. B 14, 1228 (1997).

${ }^{9}$ A. Krier, V. Sherstnev, and Y. Yakovlev, J. Appl. Phys. 33, 101 (2000).

${ }^{10}$ P. D. Altukhov, A. V. Ivanov, Y. N. Lomasov, and A. A. Rogachev, JETP Lett. 38, 4 (1983).

${ }^{11}$ P. D. Altukhov, A. M. Monakhov, A. A. Rogachev, and V. E. Khartsiev, Sov. Phys. Solid State 27, 359 (1985).

${ }^{12}$ V. M. Asnin, A. A. Rogachev, V. I. Stepanov, and A. B. Churilov, JETP Lett. 43, 365 (1986).

${ }^{13}$ V. M. Asnin, A. A. Rogachev, A. Y. Silov, and V. I. Stepanov, Solid State Commun. 74, 405 (1990).

${ }^{14}$ V. V. Sherstnev, A. M. Monahov, A. Krier, and G. Hill, Appl. Phys. Lett. 77, 3908 (2000).

${ }^{15}$ A. M. Monakhov and A. A. Rogachev, Sov. Phys. Solid State 32, 458 (1990). 
Applied Physics Letters is copyrighted by the American Institute of Physics (AIP). Redistribution of journal material is subject to the AIP online journal license and/or AIP copyright. For more information, see http:/ojps.aip.org/aplo/aplcr.jsp

Copyright of Applied Physics Letters is the property of American Institute of Physics and its content may not be copied or emailed to multiple sites or posted to a listserv without the copyright holder's express written permission. However, users may print, download, or email articles for individual use. 